\title{
TypA is involved in virulence, antimicrobial resistance and biofilm formation in Pseudomonas aeruginosa
}

\author{
Anke Neidig ${ }^{1}$, Amy TY Yeung ${ }^{2}$, Thibaut Rosay ${ }^{3}$, Beatrix Tettmann ${ }^{1}$, Nikola Strempel ${ }^{1}$, Martina Rueger ${ }^{1}$, \\ Olivier Lesouhaitier ${ }^{3}$ and Joerg Overhage ${ }^{1^{*}}$
}

\begin{abstract}
Background: Pseudomonas aeruginosa is an important opportunistic human pathogen and is extremely difficult to treat due to its high intrinsic and adaptive antibiotic resistance, ability to form biofilms in chronic infections and broad arsenal of virulence factors, which are finely regulated. TypA is a GTPase that has recently been identified to modulate virulence in enteric Gram-negative pathogens.

Results: Here, we demonstrate that mutation of typA in $P$. aeruginosa resulted in reduced virulence in phagocytic amoebae and human macrophage models of infection. In addition, the typA mutant was attenuated in rapid cell attachment to surfaces and biofilm formation, and exhibited reduced antibiotic resistance to ß-lactam, tetracycline and antimicrobial peptide antibiotics. Quantitative RT-PCR revealed the down-regulation, in a typA mutant, of important virulence-related genes such as those involved in regulation and assembly of the Type III secretion system, consistent with the observed phenotypes and role in virulence of $P$. aeruginosa.
\end{abstract}

Conclusions: These data suggest that TypA is a newly identified modulator of pathogenesis in P. aeruginosa and is involved in multiple virulence-related characteristics.

Keywords: Pseudomonas aeruginosa, Pathogen, TypA, Type III secretion system, Virulence, Dictyostelium discoideum, Macrophage, Biofilm, Resistance

\section{Background}

Pseudomonas aeruginosa is a ubiquitous environmental Gram-negative soil bacterium that is also an important opportunistic human pathogen causing a variety of different nosocomial infections including pneumonia, catheter and urinary tract infections as well as sepsis in burn wound and immunocompromised patients [1]. Moreover, $P$. aeruginosa is the most prevalent and significant pulmonary pathogen in patients with cystic fibrosis causing eventually fatal lung disease [2]. The inability to successfully clear $P$. aeruginosa infections through antibiotic treatment is a major contributor to the complicated and often severe outcome of $P$. aeruginosa infections [3]. It demonstrates high intrinsic resistance to antibiotics and

\footnotetext{
* Correspondence: joerg.overhage@kit.edu

${ }^{1}$ Karlsruhe Institute of Technology (KIT), Institute of Functional Interfaces, PO Box 3640, 76021, Karlsruhe, Germany

Full list of author information is available at the end of the article
}

an ability to develop even higher resistance through mutation, acquisition of genetic elements, and adaptation to environmental conditions, e.g. through biofilm formation on surfaces.

P. aeruginosa also possesses a large arsenal of virulencerelated factors. Among others are a type II, III and VI secretion system and their associated effector proteins such as extracellular proteases and phospholipases and the Type III secreted toxins ExoU, S, T and Y. In addition, they have flagella and type IV pili that are involved in motility and host cell adhesion [4-6]. P. aeruginosa also regulates the gene expression of most virulence factors including genes involved in iron acquisition (e.g. pyoverdine), toxin production (hydrogen cyanide), exopolysaccharide biosynthesis or biofilm formation in a cell density dependant manner termed quorum sensing mediated by the two master regulators LasR and RhlR $[4,7,8]$. Although some virulence factors seem to be host or site specific, the majority are 
involved in multi-host infections in a variety of different non-mammalian and mammalian organisms including amoebae, flies, nematodes, rodents and humans [9-11].

The coordinated control of the production of virulence and antibiotic resistance factors and the ability to adapt to various environmental changes is a likely and important reason that $P$. aeruginosa is a successful and common pathogen. The genome sequence of this microorganism revealed that more than 500 genes, representing nearly $10 \%$ of the genome, have a putative role in regulation [1]. In addition to conventional regulators involved in transcription of particular genes, e.g. sigma factors, repressors, activators or two-component response regulators, $P$. aeruginosa possesses several additional proteins that modulate translation, protein biosynthesis and degradation, etc. Here we have defined the role of the GTPase TypA in the lifestyle of P. aeruginosa.

TypA, also named BipA, belongs to a superfamily of ribosome-binding GTPases within the TRAFAC class (translation factors) of GTPases [12-14]. GTPases are widely distributed molecular switches found across all bacterial species, and generally cycle between a GDPbound "off" state and a GTP-bound "on" state $[14,15]$. Collectively they are involved in the regulation of multiple cellular processes and can play important roles in translation, ribosome biogenesis and assembly, tRNA modification, protein translocation, cell polarity, cell division and signaling events $[14,16]$. Since GTPases are widely conserved in prokaryotes and play an essential role in many important bacterial processes, they are an attractive target for novel antibiotic development [17].

TypA is highly conserved in bacteria and shares sequence homologies to other GTPases like elongation factor G. It is found in many pathogens of significant public health importance including Vibrio cholera, Yersinia pestis and Mycobacterium tuberculosis [13]. Although its precise function is still poorly understood, TypA has been suggested to be involved in the regulation of virulence and stress responses in pathogenic Escherichia coli $[18,19]$ and Salmonella enterica Serovar Typhimurium [15], and stress responses in non-pathogenic Sinorhizobium meliloti [20] and Bacillus subtilis [21]. Open reading frame PA5117 is annotated as the GTPase TypA, exhibits $75 \%$ sequence homology to TypA/BipA from $E$. coli [13], and plays a role in swarming motility and biofilm formation in $P$. aeruginosa PAO1 [22]. However, the role of TypA in pathogenesis of $P$. aeruginosa is still unknown.

Here we constructed a knock-out mutant of typ $A$ in $P$. aeruginosa PA14 and demonstrated the involvement of TypA in the pathogenesis of $P$. aeruginosa using different in vitro and in vivo infection model systems. Consistent with these data, we showed using gene expression analysis that several virulence-associated genes were down-regulated in a TypA mutant during host-pathogen interaction. We also found that TypA plays a role in antibiotic resistance to a variety of different antibiotics and initial attachment leading to subsequent biofilm formation in $P$. aeruginosa PA14.

\section{Results}

\section{TypA is involved in $P$. aeruginosa virulence}

Previously, we showed that a mutation in the typ $A$ gene led to a defect in particular virulence-associated features such as swarming and biofilm formation in P. aeruginosa PAO1 [22]. To further investigate the involvement of TypA in the pathogenesis of $P$. aeruginosa, we constructed a site-directed typA knock-out mutant in $P$. aeruginosa strain PA14. Strain PA14 is capable of infecting a wide range of organisms including the amoeba $D$. discoideum $[23,24]$ and the nematode $C$. elegans [4] and was therefore more suitable for virulence analysis using in vivo model systems in comparison to strain PAO1.

Detailed analyses of virulence attenuation of the PA14 typA mutant using the unicellular eukaryotic model organism $D$. discoideum revealed a consistent, statistically significant $(P<0.001$ by Mann Whitney test $) 2$-fold reduction in the numbers of amoebae required to form a plaque when compared to wild type strain PA14 (Figure 1). The virulence phenotype could be completely restored to wild type level by heterologous expression of the cloned typA gene in strain PA14 typA::ptyp $A^{+}$. In comparison, a similar 2 -fold reduction in numbers of amoebae was determined when analyzing PA14 transposon mutant ID29579 obtained from the Harvard PA14 mutant library [25] with a defect in the $p s c C$ gene, which is an essential part of the Type III secretion system machinery [26], as a control (Figure 1). To exclude the fact that a simple growth deficiency of the typ $A$ mutant is responsible for the attenuated virulence phenotype of PA14 typA, we performed growth analyses at $23^{\circ} \mathrm{C}$ and $37^{\circ} \mathrm{C}$ in $\mathrm{M} 9$ minimal medium using a Tecan plate reader under shaking conditions. At both temperatures no significant growth defect was observed (data not shown).

Since phagocytosis of pathogens by macrophages is a crucial factor in the human immune defense system, we quantitatively analyzed in vitro uptake of PA14 WT and respective mutant strains using human macrophages in a gentamicin protection assay. We determined a more than 2-fold increase in internalization of the typ $A$ and the psc $C$ mutant strain in comparison to cells of PA14 $\mathrm{WT}$ and complemented strain PA14 typA::ptyp $A^{+}$ (Figure 2). This result was in accord with the virulence defect observed in the amoeba model of infection, which is similarly based on phagocytic killing of bacterial cells.

To better understand the mechanism of virulence deficiency in the typA mutant, we additionally determined virulence in a nematode infection model using C. elegans as host organism under slow killing conditions. In contrast 


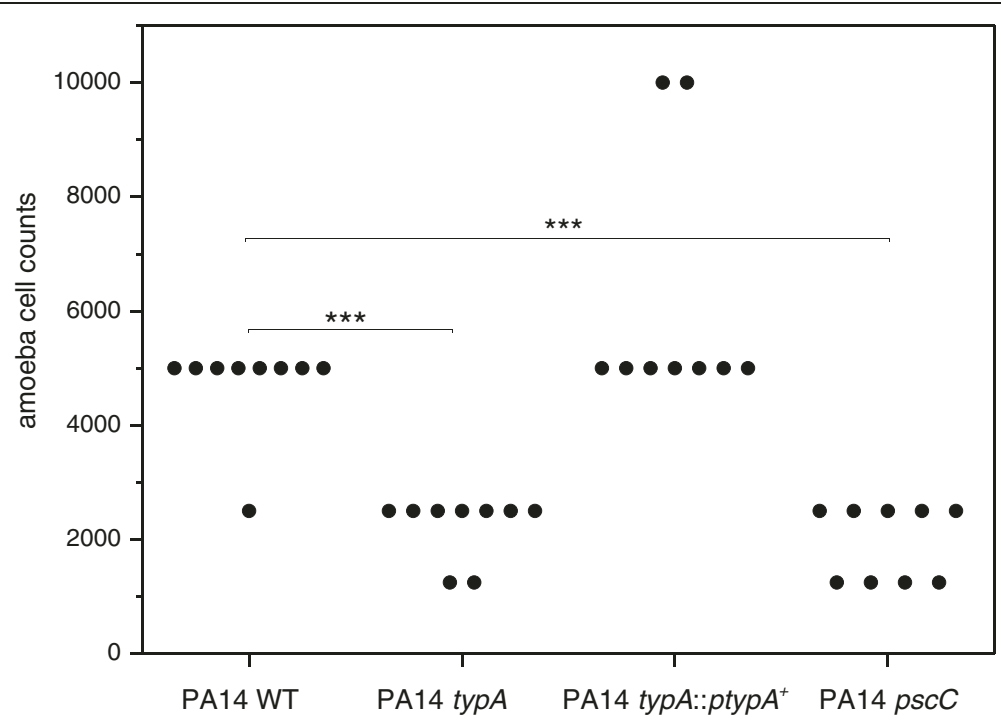

Figure $1 D$. discoideum plate killing assay. Each point represents the number of amoebae required to form a plaque on the bacterial lawn of P. aeruginosa PA14 strains after 5 days of incubation. The typA and pscC mutants had a major defect in this virulence model of infection, which was statistically significant as measured with the Mann Whitney test (*** $p<0.001, n=9$ ).

to the Type III secretion based killing of unicellular eukaryotic hosts like amoebae or macrophages, nematode killing is rather dependent on quorum sensing related virulence features in P. aeruginosa [4,27]. When feeding C. elegans with PA14 wild type, typA mutant and

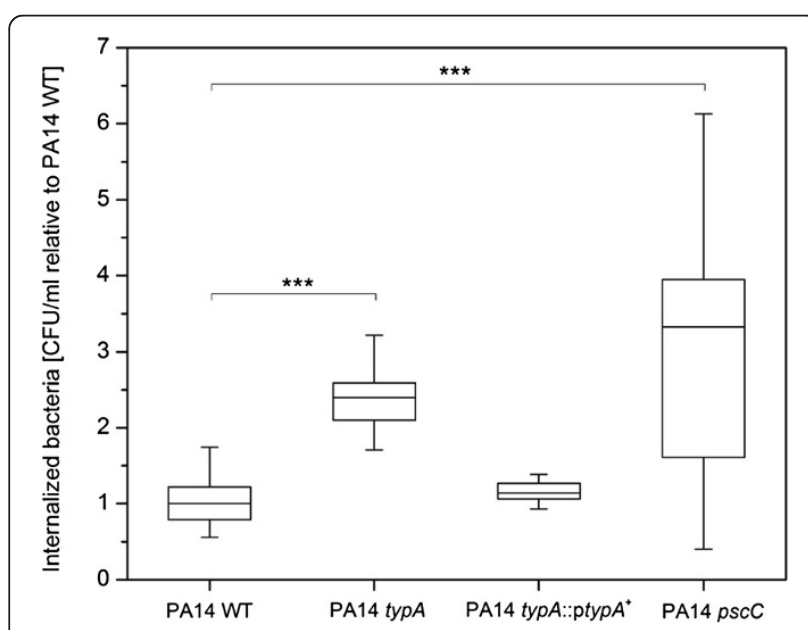

Figure 2 Uptake of $P$. aeruginosa by human macrophages. Strains were incubated with $1.5 \times 10^{5}$ cells $/ \mathrm{ml}$ macrophages for $1 \mathrm{~h}$ at an $\mathrm{MOI}$ of 10. Subsequently, extracellular and attached bacteria were killed by treatment with gentamicin, and macrophages were lysed with Triton X-100 and lysates plated to enumerate viable intracellular bacteria. Results are expressed as the percentage of intracellular bacteria that were recovered relative to the PA14 WT. The box plots (median, thick line in the box) represent the mean of 3 independent biological repeats, each assayed minimum in duplicate $(n=\geq 6) .{ }^{* *}$ indicates a statistically significant difference $(p<0.001)$, between the typA and pscC mutant and PA14 WT as determined by Whitney Mann test. complemented PA14 typA::ptyp $A^{+}$strain, we observed a similar worm killing rate for all tested strains with only marginal differences between PA14 wild type and typA knock-out mutant at day 4 of the incubation time (Figure 3).

\section{TypA is involved in rapid attachment and biofilm formation}

The ability to form biofilms is a known and important factor in the pathogenesis of $P$. aeruginosa. To assess the ability of the typA mutant to develop biofilms, static microtiter assays were performed to show that PA14 typA displayed with approximately $20 \%$ reduction a statistically

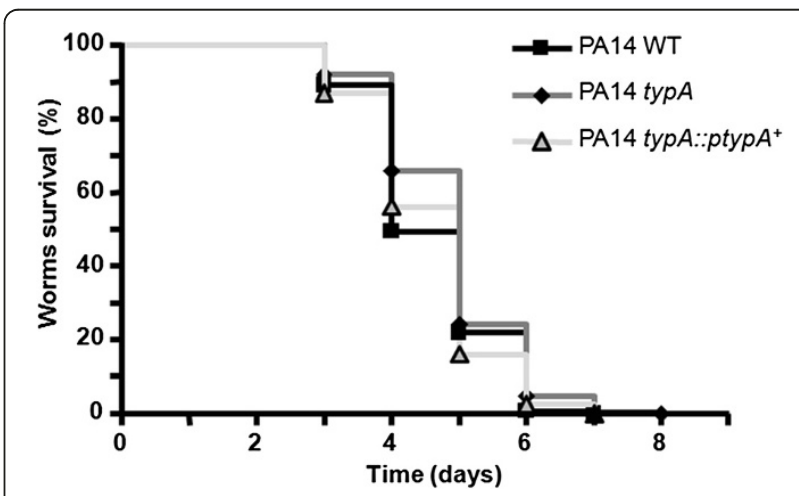

Figure $3 P$. aeruginosa virulence towards $C$. elegans worms. (a) Slow killing: Kaplan-Meier survival plots of worms fed with $P$. aeruginosa PA14 control $(n=320)$ (squares), PA14 typA mutant $(n=277)$ (diamonds) and the complemented strain PA14 typA::ptypA $A^{+}$mutant ( $n=319$ ) (triangles). Each value reported for the assay is the mean of measurements of nine samples from three independent experiments. 
significant $(P<0.001$ by Mann Whitney test) impairment in biofilm formation at 24 hours (Figure 4) in comparison to the PA14 WT. This biofilm defect could be complemented by heterologous expression of wild type typA in strain PA14 typA::ptyp $A^{+}$. To analyze whether this biofilm formation phenotype emerged during the initial adherence phase or later during biofilm growth, a rapid attachment assay was carri d out. The mutant PA14 typA exhibited with approximately $20 \%$ reduction a statistically significant $(P<0.001$ by Mann Whitney test) defect in adherence which was similar to the biofilm phenotype.

However, the investigation of flagellum-mediated swimming and swarming motility as well as the type IV pilus- mediated twitching motility, which are all involved in attachment and subsequent biofilm development, revealed no differences between mutant and wild type strain (data not shown) ruling out defects in the biosynthesis and function of these cellular appendages in the typ $A$ mutant.

\section{Antibiotic susceptibility testing}

Since recent studies have demonstrated a role for TypA in multidrug resistance in $E$. coli [28], we studied the impact of the typA gene in antibiotic resistance of $P$. aeruginosa against a variety of different antimicrobial compounds. As shown in Table 1, the typA mutant exhibited a consistent 2-fold increase in susceptibility to
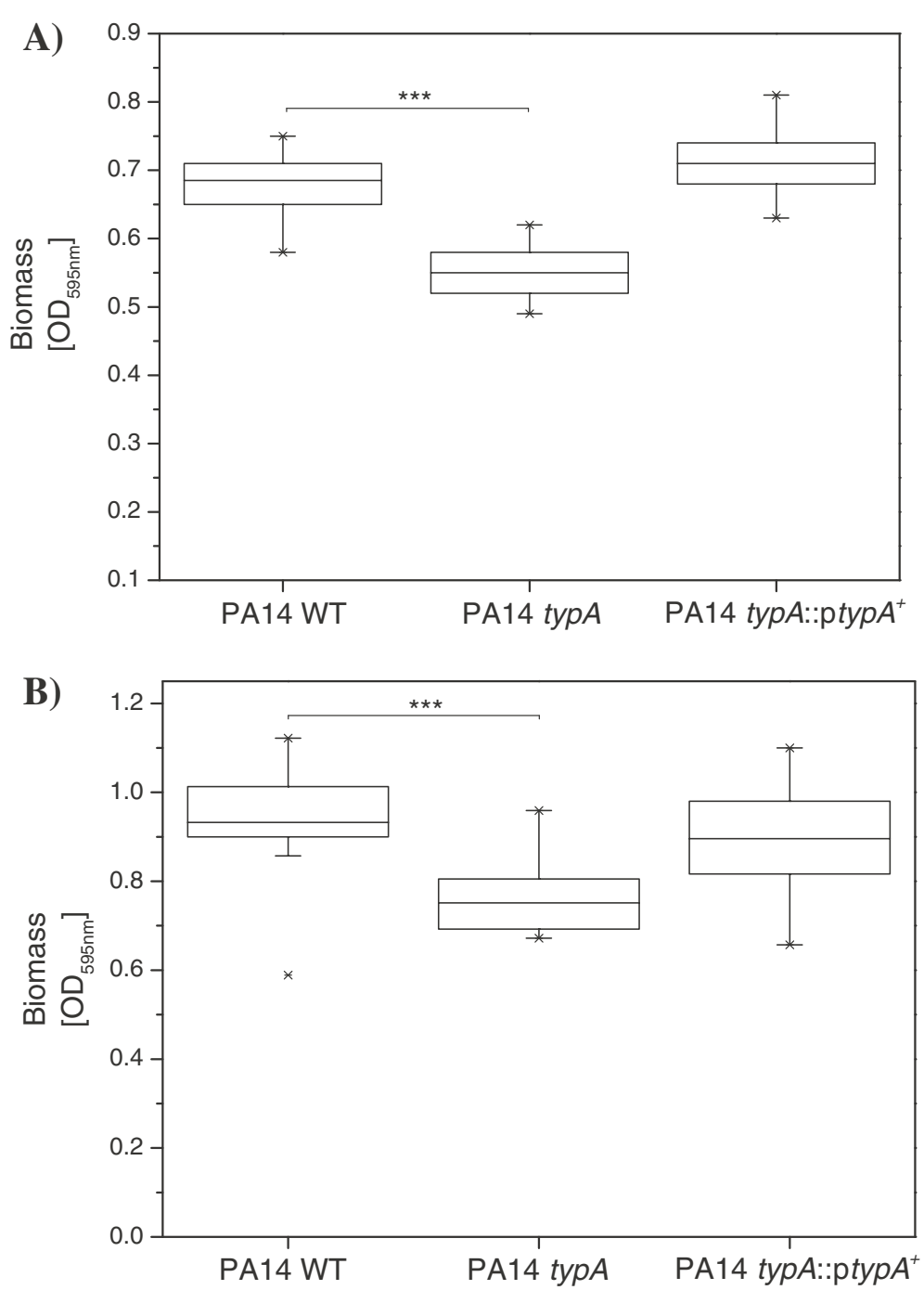

Figure 4 Defects in attachment and biofilm formation in the typA mutant. (A) Requirement for typA in rapid attachment. Attachment was determined using diluted overnight cultures for $60 \mathrm{~min}$ at $37^{\circ} \mathrm{C}$. Adhered cells were stained with crystal violet. (B) Requirement for typA in static biofilm formation. Cells were grown for $24 \mathrm{~h}$ at $37^{\circ} \mathrm{C}$ in polystyrene microtiter plates containing BM2 medium with $0.5 \%$ (w/v) casamino acids. Microtiter wells were washed several times during incubation to remove planktonic bacteria. Adherent biofilms were stained with crystal violet, followed by ethanol solubilization of the crystal violet and quantification $\left(A_{595 \mathrm{~nm}}\right)$ of stained wells. The box plots (median, thick line in the box) represent the mean of 3 independent biological repeats, each assayed in quintuplicate $(n=15)$. *** indicates a statistically significant difference $(p<0.001)$, between the typA mutant and PA14 WT as determined by Whitney Mann test. 
the cationic peptides polymyxin $\mathrm{B}$ and colistin, the $ß$ lactam antibiotics ceftazidime and meropenem, as well as tetracycline in comparison to the parent strain. This altered susceptibility could be complemented by introducing wild type copies of typA into the mutant strain. No change in susceptibility was observed regarding the fluoroquinolone ciprofloxacin, the aminoglycoside tobramycin, and the cationic host defence peptide LL-37 (Table 1).

\section{Reduced virulence of PA14 typA due to down-regulation} of the Type III secretion system

Previous studies have shown, that uptake by and killing of eukaryotic host cells is highly dependent on the Type III secretion system in $P$. aeruginosa $[5,29,30]$. To analyze the potential molecular basis for reduced virulence of the typ $A$ mutant observed in our experiments, we investigated gene expression of known virulence-associated genes in $P$. aeruginosa using qRT-PCR on bacterial RNA of wild type and typA mutant strain isolated during host-pathogen interaction with $D$. discoideum. These studies revealed that under the tested conditions, genes coding for the synthesis, function and regulation of the Type III secretion system, were significantly down-regulated in the typ $A$ mutant compared to wild type (Table 2). This observed down-regulation of important virulence-related genes is consistent with the noticed virulence defects in the cellular infection studies with $D$. discoideum and human macrophages as hosts.

\section{Discussion}

In this study, we have shown that TypA is involved in virulence of $P$. aeruginosa by analyzing the consequences of a typA knock-out on phagocytic amoebae and human macrophages as well as the interaction with the

\begin{tabular}{|c|c|c|c|}
\hline \multirow[b]{2}{*}{ Antibiotic } & \multicolumn{3}{|c|}{$\mathrm{MIC}(\mu \mathrm{g} / \mathrm{ml})$} \\
\hline & PA14 WT & PA14 typA & PA14 typA::ptypA ${ }^{+}$ \\
\hline Ciprofloxacin & 0.03 & 0.03 & 0.03 \\
\hline Meropenem & 2 & 1 & 2 \\
\hline Ceftazidime & 4 & 2 & 4 \\
\hline Tetracycline & 8 & 4 & 8 \\
\hline Tobramycin & 0.25 & 0.25 & 0.25 \\
\hline Polymyxin B & 0.5 & 0.25 & 0.5 \\
\hline Colistin & 0.25 & 0.125 & 0.25 \\
\hline LL-37 & 16 & 16 & 16 \\
\hline
\end{tabular}

${ }^{\mathrm{a}} \mathrm{MICs}$ were determined by serial 2-fold dilutions in $\mathrm{MH}$-medium. The MIC represents the concentration at which no growth was visually observed after $24 \mathrm{~h}$ of incubation at $37^{\circ} \mathrm{C}$. The values shown are the modes of 4 to 6 independent experiments.
Table 2 Gene expression of selected Type III secretion genes in the typA mutant compared to that in wild type PA14 using RT-qPCR

\begin{tabular}{lc}
\hline Gene & $\begin{array}{c}\text { Fold change in gene expression in the typA mutant } \\
\text { relative to wild type }{ }^{\mathbf{a}}\end{array}$ \\
\hline T3SS & \\
exsA & $-3.1 \pm 0.5$ \\
psCC & $-2.3 \pm 0.4$ \\
psCJ & $-3.5 \pm 0.3$ \\
psCT & $-5.1 \pm 0.3$ \\
pCrV & $-5.8 \pm 0.6$ \\
\hline
\end{tabular}

nematode $C$. elegans. Moreover, TypA also contributes to resistance to different antibiotics as well as attachment and biofilm formation in $P$. aeruginosa.

TypA is a highly conserved prokaryotic GTPase exhibiting structural homologies to translation factor GTPases such as EF-G and LepA and is described to associate with the ribosomes under normal bacterial growth [15,31]. In enteropathogenic E. coli (EPEC), TypA co-ordinates the expression of key stress and virulence factors including flagella, Type III secretion system as well as the LEE and the espC pathogenicity islands $[18,32]$ by regulating gene expression of major regulators such as Ler, which in turn controls these respective pathogenicity islands. Consequently, it has been suggested that TypA is on a relatively high level in the complex regulatory hierarchy of virulence regulation in this organism [18,32]. In contrast, analysis in Mycobacterium tuberculosis revealed that TypA does not act as a virulence regulator in this human pathogen, ruling out a general involvement of this protein in virulence regulation in pathogenic bacteria [33]. However, our results demonstrate that TypA plays a role in the pathogenesis of $P$. aeruginosa. The typA knock-out mutant exhibited a significant virulence deficiency in both the amoebae infection model and the macrophage uptake studies. These defects were comparable to a $p s c C$ mutant with a disrupted Type III secretion system and consistent with the down-regulation of Type III secretion genes during host-pathogen interaction. The Type III secretion system of Gram-negative bacteria is an important factor of pathogenesis and is involved in manipulating eukaryotic cells by injecting effector proteins into the host [27] and impacts diretly on bacterial uptake by phagocytic cells [30]. In $P$. aeruginosa, this complex, needle-like machinery is encoded by 36 genes and an important factor for the survival during interaction with phagocytic amoebae or human macrophages, among others [5,29,30]. Using qRT-PCR, we observed the down-regulation of selected genes participating in different aspects of Type III secretion, including $p s c C$ (outer membrane ring), pscJ (basal substructure), $p s c T$ (translocation), and $p c r V$ (needle-tip 
complex). The transcription of Type III secretion genes is tightly regulated by ExsA in $P$. aeruginosa. This master regulator controls both, the synthesis of the secretion system as well as effector protein production, and interacts in concert with the global cyclic AMP and Gac regulatory systems [5,34]. Our studies showed that in addition to genes involved in assembly of the secretion apparatus, expression of exsA was also significantly down-regulated in the typ $A$ mutant compared to wild type cells. To identify, if increasing Type III secretion activity is sufficient to complement our virulence phenotype, we heterologously expressed the exs $A$ gene using plasmid pUCP20::exs $A^{+}$in the typ $A$ mutant and obtained an identical number of amoebae required for plaque formation in both mutant and wild type PA14 harboring pUCP20::exsA (data not shown). These findings suggest that, like in E. coli, TypA is part of the complex regulatory cascade involved in controlling Type III secretion in $P$. aeruginosa by impacting expression of genes involved in regulation and assembly of the secretion machinery. Since TypA is a GTPase associated with the ribosomes, a further down-regulation of the Type III secretion machinery at the translational level might also be possible; this could result in an even stronger impairment of the Type III secretion system.

Previously, it has been shown that the Type III secretion system including its associated virulence effectors does not play a noticeable role in nematode killing $[4,35]$, which is rather dependent on quorum sensing related virulence factors such as RhlR and LasR $[27,36]$. Thus, it is not surprising, that a mutation in typ $A$ with a down-regulation in the Type III secretion system did not result in significant virulence attenuation in our studied infection model. Additional analyses of quorum sensing dependent production of the extracellular protease LasB and toxin pyocyanin did not reveal a significant difference between wild type and mutant strain (data not shown) demonstrating that TypA does, most likely, not affect quorum sensing in $P$. aeruginosa PA14.

TypA was first described to be involved in human bactericidal/permeability-increasing protein BPI, a cationic host defence peptide from human neutrophils, resistance in S. typhimurium and E. coli $[37,38]$. Although we were not able to detect any differences regarding resistance to cationic human host defence peptide LL-37, we found that TypA is also participating in resistance against a variety of clinically important antibiotics such as ß-lactam, tetracycline and peptides antibiotics in $P$. aeruginosa. Due to this wide range of different antimicrobials with unrelated modes of action, it is likely that the involvement of TypA in antibiotic stress resistance is rather unspecific and could be based on the fact that TypA is part of a more general stress response resulting in resistance. This would be in accordance with earlier studies showing the involvement of TypA in a wide variety of very different stress responses in a number of pathogenic and nonpathogenic microorganisms, among other stress factors were antimicrobials, low $\mathrm{pH}$, oxidative or detergent stress $[20,37,38]$.

Biofilm formation is a crucial factor in the pathogenesis of $P$. aeruginosa and is involved in many chronic infections including chronic lung infections of cystic fibrosis patients or foreign body part infections [39]. Biofilm development is a sequential process initiated by the attachment of planktonic cells to a surface, followed by formation of microcolonies and biofilm maturation. Bacteria grown in biofilms exhibit high resistance against antimicrobial agents, are protected from the host immune response and are notoriously difficult to eradicate [39-41]. Although the typA mutant was able to form biofilms, we observed a more than $20 \%$ reduction in biofilm mass compared to wild type cells. By analyzing the initial adhesion phase of biofilm development, we identified that this reduction in biofilm is, at least in parts, due to a significant impairment in rapid attachment of the typA mutant in the respective microtiter plate assay. This impairment in attachment results in less bacterial cells initiating biofilm formation and subsequently lower biofilm growth, which could not be restored to wild type levels during further biofilm development. Interestingly, it was shown previously that TypA is involved in adherence to biotic surfaces and interaction of enteropathogenic E. coli with epithelial cells [19] and the symbiotic interaction of $S$. meliloti with the nodules of the legume Medicago truncatula [20] indicating a role of TypA in cell-cell contact. Biofilm initiation and cell adhesion are rather complex processes influenced by a large number of proteins and factors, among others are flagellum- and type IV pilus-mediated bacterial motility and attachment, respectively. Although we have recently shown, that TypA is involved in swarming motility in $P$. aeruginosa strain PAO1 [22], we did not observe any impairment in swimming, swarming or twitching motility in the PA14 typA mutant suggesting a mechanism not related to a defect in flagella or type IV pili biogenesis and function, respectively, is responsible for the impairment in adhesion and biofilm initiation in this mutant.

\section{Conclusions}

In this study, we were able to demonstrate the involvement of TypA in the pathogenesis of $P$. aeruginosa by analyzing the consequences of a typA knock-out. This typ $A$ mutant exhibited reduced virulence towards phagocytic amoebae and increased uptake by human macrophages, impaired cell attachment and subsequent biofilm formation and a reduction in antimicrobial resistance to $\beta$ lactam, tetracycline and antimicrobial peptide antibiotics. The typA mutant exhibited a dysregulation of genes 
involved in regulation and assembly of the Type III secretion system, consistent with the observed phenotypes and role in virulence regulating Type III secretion system.

\section{Methods}

\section{Organisms, plasmids, primers, and growth conditions}

The organisms and plasmids used in this study are listed in Table 3 and include P. aeruginosa PA14 [25] and Dictyostelium discoideum Ax2 [24]. The sequences of DNA primers (Eurofins MWG Operon, Germany) used in these studies are available upon request. E. coli was routinely grown in Luria-Bertani (LB) broth, $P$. aeruginosa in M9 [23], LB or BM2 [44] medium, and $D$. discoideum in HL5 broth medium [45]. D. discoideum was incubated in cell culture flasks (Greiner Bio One, Frickenhausen, Germany) at $22.5^{\circ} \mathrm{C}$ and sub-cultured twice a week. When required for plasmid or resistance gene selection or maintenance, gentamicin, ampicillin and carbenicillin were added at final concentrations of 30,100 and $200 \mu \mathrm{g} / \mathrm{ml}$, respectively.

\section{Amoeba plaque assay}

In this cellular model system, a more virulent $P$. aeruginosa strain will limit the ability of the amoebae to form a plaque on a bacterial lawn to a greater extent than a less virulent strain. The assay was performed according to the method described previously [23]. Briefly, $50 \mu \mathrm{l}$ of overnight cultures grown in LB medium were mixed with $200 \mu \mathrm{l}$ PBS buffer and plated on M9 agar plates. Plates were dried on a laminar flow bench for 15 min to obtain a dry, even bacterial lawn. Amoebae grown for 2 to 4 days in the respective medium were harvested by centrifugation at $510 \mathrm{x}$ g for 10 minutes, washed and resuspended in PBS buffer. Cells were adjusted to $8 \times 10^{6}$ cells per $\mathrm{ml}$ and kept on ice.
This stock solution was serially diluted and used to prepare droplets of $5 \mu \mathrm{l}$ containing between 5 and 20,000 amoebae, which were subsequently spotted onto the bacterial lawn. Plates were incubated for 5 days at $22.5^{\circ} \mathrm{C}$ and the highest dilution at which growth of the amoebae caused a visible plaque of bacterial clearance was reported. Three independent experiments performed at least in duplicate were carried out for each bacterial strain.

\section{Gentamicin protection assay}

In vitro internalization of PA14 WT and mutant cells by human macrophages derived from monocytes (MDM) was performed as previously described [46] with modifications. Briefly, mid-logarithmic phase cultures of $P$. aeruginosa were washed with complete RPMI medium and resuspended in $1 \mathrm{ml}$ of the medium. The resuspended bacteria were added to $1.5 \times 10^{5} \mathrm{MDM}$ cells $/ \mathrm{ml}$, at a multiplicity of infection (MOI) of 10, and incubated for $1 \mathrm{~h}$ at $37^{\circ} \mathrm{C}$. Subsequently, cells were washed with complete RPMI and incubated with $400 \mu \mathrm{g} / \mathrm{ml}$ of gentamicin for $30 \mathrm{~min}$ at $37^{\circ} \mathrm{C}$ to kill the extracellular and attached bacteria. After gentamicin treatment, MDM cells were washed and lysed with $0.1 \%$ Triton X-100. Lysates were plated onto LB agar and incubated overnight at $37^{\circ} \mathrm{C}$. The next day, colonies were counted and relative phagocytic uptake was determined by CFU counts. Three independent experiments with at least duplicates in each experiment were performed for each bacterial strain.

\section{Caenorhabditis elegans synchronization and virulence assay} The C. elegans wild-type Bristol strain N2 was obtained from the Caenorhabditis Genetics Center (Minneapolis, MN, USA). C. elegans were maintained under standard culturing conditions at $22^{\circ} \mathrm{C}$ on nematode growth

Table 3 Strains and plasmids used in this study

\begin{tabular}{|c|c|c|}
\hline $\begin{array}{l}\text { Strain or plasmid } \\
\text { Strains }\end{array}$ & Strains & Reference \\
\hline \multicolumn{3}{|l|}{ P. aeruginosa } \\
\hline PA14 WT & Wild type $P$. aeruginosa PA14 & [25] \\
\hline PA14 typA & typA insertion mutant of PA14, $\mathrm{Gm}^{r}$ & This study \\
\hline PA14 typA::ptypA ${ }^{+}$ & Complemented mutant PA14 typA harboring plasmid pUCP20:typA $A^{+} ; \mathrm{Gm}^{r}, \mathrm{Cb}^{r}$ & This study \\
\hline PA14 pscC & pscC transposon mutant ID29579 of the Harvard PA14 mutant library & [25] \\
\hline \multicolumn{3}{|l|}{ E. coli } \\
\hline $\mathrm{DH} 5 \mathrm{a}$ & 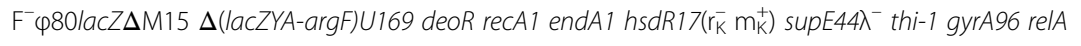 & Invitrogen \\
\hline \multicolumn{3}{|l|}{ Plasmids } \\
\hline pUCP20 & E. coli - Pseudomonas shuttle vector for constitutive expression of cloned genes, $\mathrm{Cb}^{r}$ & [42] \\
\hline pEX18Ap & Suicide vector for mutant regeneration in Pseudomonas, $\mathrm{Amp}^{\mathrm{r}} / \mathrm{Cb}^{\mathrm{r}}$ & [43] \\
\hline pUCP20::typ $A^{+}$ & pUCP20 containing the cloned typA gene; $\mathrm{Amp}^{r} / \mathrm{Cb}^{r}$ & This study \\
\hline pUCP20::exs $A^{+}$ & pUCP20 containing the cloned exs $\mathrm{A}$ gene; $\mathrm{Amp}^{r} / \mathrm{Cb}^{r}$ & This study \\
\hline
\end{tabular}

${ }^{a}$ Antibiotic resistance phenotypes: Ampr ${ }^{r}$, ampicillin resistance for $E . \mathrm{Coli} \mathrm{Cb}^{r}$, carbenicillin resistance for $P$. aeruginosa; $\mathrm{Gm}^{r}$, gentamicin resistance. 
medium (NGM: $3 \mathrm{~g} \mathrm{NaCl}, 2.5 \mathrm{~g}$ peptone, $17 \mathrm{~g}$ agar, $5 \mathrm{mg}$ cholesterol, $1 \mathrm{ml} 1 \mathrm{M} \mathrm{CaCl}_{2}, 1 \mathrm{ml} 1 \mathrm{M} \mathrm{MgSO}_{4}, 25 \mathrm{ml}$ $1 \mathrm{M} \mathrm{KH}_{2} \mathrm{PO}_{4}, \mathrm{H}_{2} \mathrm{O}$ to 1 liter) agar plates with $E$. coli OP50 as a food source [47]. Synchronous cultures of worms were generated after worm adult population exposure to a sodium hypochlorite/sodium hydroxide solution as previously described [48] and adapted [49]. The resulting eggs were incubated at $22^{\circ} \mathrm{C}$ on an E. coli OP50 lawn until the worms reached the L4 (48 hours) life stage (confirmed by light microscopy). Bacterial lawns used for $C$. elegans survival assays were prepared by spreading $50 \mu \mathrm{l}$ of $P$. aeruginosa strains on $35 \mathrm{~mm}$ NGM conditioned Petri dishes supplemented with $0.05 \mathrm{mg} \mathrm{ml-}{ }^{1}$ 5-fluoro-2'-deoxyuridine. This nucleotide analog blocks the development of the next C. elegans generation by inhibition of DNA synthesis. The plates were incubated overnight at $37^{\circ} \mathrm{C}$ and then placed at room temperature for $4 \mathrm{~h}$. Fifteen to twenty L4 synchronized worms were harvested by resuspension in M9 buffer (3 g K $\mathrm{KH}_{2} \mathrm{PO}_{4}, 6$ g NaHPO $4,5 \mathrm{~g} \mathrm{NaCl}, 1 \mathrm{ml} 1 \mathrm{M}$ $\mathrm{MgSO}_{4}, \mathrm{H}_{2} \mathrm{O}$ to 1 liter), plated on the $35 \mathrm{~mm}$ assay Petri dishes and incubated at $22^{\circ} \mathrm{C}$. Worm survival was scored after $1 \mathrm{~h}, 24 \mathrm{~h}$ and on each subsequent day, using an Axiovert S100 optical microscope (Zeiss, Oberkochen, Germany) equipped with a Nikon digital Camera DXM 1200 F (Nikon Instruments, Melville, NY, USA). Worms were considered dead when they remained static without grinder movements for $20 \mathrm{~s}$. The results were expressed as the percentage of living worms and were the average of three independent assays performed in triplicate.

\section{Growth curves}

Overnight cultures grown in LB medium were diluted into M9 medium to obtain equal starting optical densities at $600 \mathrm{~nm}\left(\mathrm{OD}_{600}\right)$. Five- $\mu \mathrm{l}$ portions of these cultures were added to $195 \mu \mathrm{l}$ of fresh M9 medium in 96-well microtiter plates. The growth of the cultures at $37^{\circ} \mathrm{C}$ and $23^{\circ} \mathrm{C}$ under shaking conditions was monitored with a Tecan Infinite F200 Pro.

\section{Plasmid and typA knock-out mutant generation}

For the construction and complementation of a typ $A$ knock-out mutant in $P$. aeruginosa PA14 the typA gene (gene number PA_67560) was amplified by PCR using EcoRI and HindIII flanked oligonucleotides, respectively, and subsequently cloned behind the lac promoter in the broad host range vector pUCP20, resulting in pUCP20:: $\operatorname{typ} A^{+}$. For the heterologous expression of the exs $A$ gene, exsA was amplified by PCR using EcoRI and XbaI flanked oligonucleotides, respectively, and subsequently cloned into pUCP20, resulting in pUCP20::exs $A^{+}$. These plasmids were then transferred into $E$. coli $\mathrm{DH} 5 \alpha$ by transformation or $P$. aeruginosa by electroporation. The knock-out mutant was obtained according to the methods described previously [43]. Briefly, the hybrid plasmid pUCP20::typ $A^{+}$was digested with SmaI to delete a $1.1 \mathrm{~kb}$ fragment from the typ $A$ gene, which was subsequently replaced with a $\Omega$ gentamicin resistance gene cassette for selection. The disrupted typ $A \Omega \mathrm{Gm}$ gene was amplified by PCR and cloned into the suicide vector pEX18Ap [43] and transferred into $P$. aeruginosa PA14 to generate the typA knock-out mutant named $P$. aeruginosa PA14 typA by allelic exchange.

\section{MIC determination}

MICs were measured using standard broth microdilution procedures [50] in Mueller Hinton ( $\mathrm{MH})$ medium. Growth was scored following $24 \mathrm{~h}$ of incubation at $37^{\circ} \mathrm{C}$.

\section{Motility, biofilm and rapid attachment assays}

Swimming, swarming and twitching motility were evaluated as described previously [44]. The abiotic solid surface assay was used to measure biofilm formation according to the previously described method with the following modifications [51]. Overnight cultures were diluted 1:100 in BM2 containing 0.5\% (w/v) casamino acids and inoculated into 96 -well polystyrene microtiter plates and incubated at $37^{\circ} \mathrm{C}$ for $60 \mathrm{~min}$ without shaking to allow bacterial cell adhesion. Subsequently, the microtiter wells were washed twice to remove planktonic cells and new biofilm growth medium was added. This washing step was repeated after 4 and 16 hours of incubation. After $24 \mathrm{~h}$, the biofilm was staining using crystal violet and the absorbance was measured at $595 \mathrm{~nm}$ using a Tecan Infinite F200 Pro.

Rapid attachment of bacterial cells to a surface was analyzed as described previously [44]. Briefly, overnight cultures grown in BM2-medium were washed and diluted in BM2 medium containing $0.1 \%(\mathrm{w} / \mathrm{v})$ casamino acids (CAA) to an $\mathrm{OD}_{595 \mathrm{~nm}}$ of 1.0. One hundred $\mu \mathrm{l}$ of this suspension was used to inoculate each well of a microtiter plate. Cells were allowed to adhere for $60 \mathrm{~min}$ at $37^{\circ} \mathrm{C}$ prior to staining with crystal violet.

\section{RNA extraction, CDNA synthesis, and quantitative real- time PCR (qRT-PCR)}

For analysis of virulence gene expression, overnight cultures of $P$. aeruginosa PA14 WT and the typA mutant were washed twice and resuspended in PBS buffer and adjusted to an $\mathrm{OD}_{600}$ of 2.0. For each bacterial cell suspension, $10 \mu \mathrm{l}$ was mixed with washed amoeba cells of 2-day old $D$. discoideum cultures at a ratio of 3:1 bacteria to amoebae and the mixtures were plated on M9 agar plates. After incubation for $48 \mathrm{~h}$ at $22.5^{\circ} \mathrm{C}$, cells were harvested from the agar plate surface, using an inoculation loop, and were resuspended in M9 medium supplemented with RNA protect reagent (Qiagen, Germany). To separate cells of $D$. discoideum from the bacterial cells, the 
mixtures were centrifuged for $1 \mathrm{~min}$ at 1,000 rpm and the supernatants containing the bacterial cells were used for RNA extraction.

RNA isolation, cDNA synthesis, and qRT-PCR analysis were performed as described previously [52] using the Power SYBR Green PCR Master Mix in an Abi 7300 Real Time PCR System (Applied Biosystems). All reactions were normalized to the house keeping gene rpsL. Experiments were repeated with three independent cultures.

\section{Competing interests}

The authors declare that they have no competing interest.

\section{Authors' contributions}

$\mathrm{OL}$ and $\mathrm{JO}$ designed the experiments, supervised the research and wrote the paper. AN, ATYY, TR, BT, NS and MR did experiments and/or data analysis. All authors read and approved the final manuscript.

\section{Acknowledgements}

We gratefully acknowledge financial support by the Biolnterfaces (BIF) Program of the Karlsruhe Institute of Technology (KIT) in the Helmholtz Association and by the "Concept for the Future" of the Karlsruhe Institute of Technology (KIT) within the German Excellence Initiative. ATYY received studentships from Cystic Fibrosis Canada and the Natural Sciences and Engineering Research Council of Canada (NSERC). We thank Prof. M. Steinert for kindly providing D. discoideum, Prof. G. Hänsch for help with the gentamicin protection assay, and Olivier Maillot and Magalie Barreau for technical assistance.

\section{Author details}

${ }^{1}$ Karlsruhe Institute of Technology (KIT), Institute of Functional Interfaces, PO Box 3640, 76021, Karlsruhe, Germany. ${ }^{2}$ Centre for Microbial Diseases \& Immunity Research, University of British Columbia, 2259 Lower Mall, Vancouver, BC, Canada. ${ }^{3}$ Laboratory of Microbiology Signals and Microenvironment, LMSM EA 4312, University of Rouen, 55 rue Saint Germain, 27000, Evreux, France.

Received: 30 October 2012 Accepted: 4 April 2013

Published: 9 April 2013

\section{References}

1. Stover CK, Pham XQ, Erwin AL, Mizoguchi SD, Warrener P, Hickey MJ, Brinkman FS, Hufnagle WO, Kowalik DJ, Lagrou M, et al: Complete genome sequence of Pseudomonas aeruginosa PAO1, an opportunistic pathogen. Nature 2000, 406(6799):959-964.

2. Govan JR, Deretic V: Microbial pathogenesis in cystic fibrosis: mucoid Pseudomonas aeruginosa and Burkholderia cepacia. Microbiol Rev 1996, 60(3):539-574

3. Breidenstein EB, de la Fuente-Nunez C, Hancock RE: Pseudomonas aeruginosa: all roads lead to resistance. Trends Microbiol 2011, 19(8):419-426.

4. Feinbaum RL, Urbach JM, Liberati NT, Djonovic S, Adonizio A, Carvunis AR, Ausubel FM: Genome-wide identification of pseudomonas aeruginosa virulence-related genes using a caenorhabditis elegans infection model. PLoS Pathog 2012, 8(7):e1002813.

5. Hauser AR: The type III secretion system of Pseudomonas aeruginosa: infection by injection. Nat Rev Microbio/ 2009, 7(9):654-665

6. Filloux A: Protein secretion systems in pseudomonas aeruginosa: an essay on diversity, evolution, and function. Front Microbiol 2011, 2:155.

7. Girard G, Bloemberg GV: Central role of quorum sensing in regulating the production of pathogenicity factors in Pseudomonas aeruginosa. Future Microbiol 2008, 3(1):97-106.

8. Smith RS, Iglewski BH: $P$. aeruginosa quorum-sensing systems and virulence. Curr Opin Microbiol 2003, 6(1):56-60.

9. Aballay A, Ausubel FM: Caenorhabditis elegans as a host for the study of host-pathogen interactions. Curr Opin Microbiol 2002, 5(1):97-101.

10. Lima WC, Lelong E, Cosson P: What can Dictyostelium bring to the study of Pseudomonas infections? Semin Cell Dev Biol 2011, 22(1):77-81.
11. Limmer S, Quintin J, Hetru C, Ferrandon D: Virulence on the fly: drosophila melanogaster as a model genetic organism to decipher host-pathogen interactions. Curr Drug Targets 2011, 12(7):978-999.

12. Wang F, Zhong NQ, Gao P, Wang GL, Wang HY, Xia GX: SsTypA1, a chloroplast-specific TypA/BipA-type GTPase from the halophytic plant Suaeda salsa, plays a role in oxidative stress tolerance. Plant Cell Environ 2008, 31(7):982-994.

13. Scott K, Diggle MA, Clarke SC: TypA is a virulence regulator and is present in many pathogenic bacteria. Br J Biomed Sci 2003, 60(3):168-170.

14. Verstraeten N, Fauvart M, Versees W, Michiels J: The universally conserved prokaryotic GTPases. Microbiol Mol Biol Rev 2011, 75(3):507-542. second and third pages of table of contents.

15. DeLivron MA, Robinson VL: Salmonella enterica serovar Typhimurium BipA exhibits two distinct ribosome binding modes. J Bacteriol 2008, 190(17):5944-5952

16. Britton RA: Role of GTPases in bacterial ribosome assembly. Annu Rev Microbiol 2009, 63:155-176.

17. Hwang J, Tseitin V, Ramnarayan K, Shenderovich MD, Inouye M: Structurebased design and screening of inhibitors for an essential bacterial GTPase, Der. J Antibiot (Tokyo) 2012, 65(5):237-243.

18. Grant AJ, Farris M, Alefounder P, Williams PH, Woodward MJ, O'Connor CD: Co-ordination of pathogenicity island expression by the BipA GTPase in enteropathogenic Escherichia coli (EPEC). Mol Microbiol 2003, 48(2):507-521.

19. Farris M, Grant A, Richardson TB, O'Connor CD: BipA: a tyrosinephosphorylated GTPase that mediates interactions between enteropathogenic Escherichia coli (EPEC) and epithelial cells. Mol Microbiol 1998, 28(2):265-279.

20. Kiss $E$, Huguet $T$, Poinsot $V$, Batut J: The typA gene is required for stress adaptation as well as for symbiosis of Sinorhizobium meliloti 1021 with certain Medicago truncatula lines. Mol Plant Microbe Interact 2004, 17(3):235-244.

21. Beckering CL, Steil L, Weber MH, Volker U, Marahiel MA: Genomewide transcriptional analysis of the cold shock response in Bacillus subtilis. J Bacteriol 2002, 184(22):6395-6402.

22. Overhage J, Lewenza S, Marr AK, Hancock RE: Identification of genes involved in swarming motility using a Pseudomonas aeruginosa PAO1 mini-Tn5-lux mutant library. J Bacteriol 2007, 189(5):2164-2169.

23. Breidenstein EBM L, Janot J, Strehmel L, Fernandez PK, Taylor I, KukavicaIbrulj SL, Gellatly RC, Levesque J, Overhage LJ, Hancock REW: The Lon protease is essential for full virulence in Pseudomonas aeruginosa. PLoS One 2012, 7(11):e49123.

24. Adamek M, Overhage J, Bathe S, Winter J, Fischer R, Schwartz T: Genotyping of environmental and clinical Stenotrophomonas maltophilia isolates and their pathogenic potential. PLoS One 2011, 6(11):e27615.

25. Liberati NT, Urbach JM, Miyata S, Lee DG, Drenkard E, Wu G, Villanueva J, Wei T, Ausubel FM: An ordered, nonredundant library of Pseudomonas aeruginosa strain PA14 transposon insertion mutants (vol 103, pg 2833, 2006). P Natl Acad Sci USA 2006, 103(52):19931-19931.

26. Saliba AM, Filloux A, Ball G, Silva ASV, Assis MC, Plotkowski MC: Type III secretion-mediated killing of endothelial cells by Pseudomonas aeruginosa. Microb Pathogenesis 2002, 33(4):153-166.

27. Tan MW, Rahme LG, Sternberg JA, Tompkins RG, Ausubel FM: Pseudomonas aeruginosa killing of Caenorhabditis elegans used to identify P. aeruginosa virulence factors. P Natl Acad Sci USA 1999, 96(5):2408-2413.

28. Duo M, Hou S, Ren D: Identifying Escherichia coli genes involved in intrinsic multidrug resistance. App/ Microbiol Biotechnol 2008, 81(4):731-741

29. Matz C, Moreno AM, Alhede M, Manefield M, Hauser AR, Givskov M, Kjelleberg S: Pseudomonas aeruginosa uses type III secretion system to kill biofilm-associated amoebae. ISME J 2008, 2(8):843-852.

30. Aiello D, Williams JD, Majgier-Baranowska H, Patel I, Peet NP, Huang J, Lory $\mathrm{S}$, Bowlin TL, Moir DT: Discovery and characterization of inhibitors of Pseudomonas aeruginosa type III secretion. Antimicrob Agents Chemother 2010, 54(5):1988-1999.

31. DeLivron MA, Makanji HS, Lane MC, Robinson VL: A novel domain in translational GTPase BipA mediates interaction with the 70S ribosome and influences GTP hydrolysis. Biochemistry 2009, 48(44): 10533-10541. 
32. Sircili MP, Walters $M$, Trabulsi $L R$, Sperandio V: Modulation of enteropathogenic Escherichia coli virulence by quorum sensing. Infect Immun 2004, 72(4):2329-2337.

33. Micklinghoff JC, Schmidt M, Geffers R, Tegge W, Bange FC: Analysis of expression and regulatory functions of the ribosome-binding protein TypA in Mycobacterium tuberculosis under stress conditions. Arch Microbiol 2010, 192(6):499-504.

34. Yahr TL, Wolfgang MC: Transcriptional regulation of the Pseudomonas aeruginosa type III secretion system. Mol Microbiol 2006, 62(3):631-640.

35. Wareham DW, Papakonstantinopoulou A, Curtis MA: The Pseudomonas aeruginosa PA14 type III secretion system is expressed but not essential to virulence in the Caenorhabditis elegans-P. aeruginosa pathogenicity model. FEMS Microbiol Lett 2005, 242(2):209-216.

36. Darby C, Cosma CL, Thomas JH, Manoil C: Lethal paralysis of Caenorhabditis elegans by Pseudomonas aeruginosa. P Natl Acad Sci USA 1999, 96(26):15202-15207.

37. Qi SY, Li Y, Szyroki A, Giles IG, Moir A, O'Connor CD: Salmonella typhimurium responses to a bactericidal protein from human neutrophils. Mol Microbiol 1995, 17(3):523-531.

38. Barker HC, Kinsella N, Jaspe A, Friedrich T, O'Connor CD: Formate protects stationary-phase Escherichia coli and Salmonella cells from killing by a cationic antimicrobial peptide. Mol Microbiol 2000, 35(6):1518-1529.

39. Hoiby N, Ciofu O, Johansen HK, Song ZJ, Moser C, Jensen PO, Molin S, Givskov M, Tolker-Nielsen T, Bjarnsholt T: The clinical impact of bacterial biofilms. Int J Oral Sci 2011, 3(2):55-65.

40. Jensen PO, Givskov M, Bjarnsholt T, Moser C: The immune system vs Pseudomonas aeruginosa biofilms. FEMS Immunol Med Microbiol 2010, 59(3):292-305

41. Mah TF, OToole GA: Mechanisms of biofilm resistance to antimicrobial agents. Trends Microbiol 2001, 9(1):34-39.

42. West SE, Schweizer HP, Dall C, Sample AK, Runyen-Janecky LJ: Construction of improved Escherichia-Pseudomonas shuttle vectors derived from pUC18/19 and sequence of the region required for their replication in Pseudomonas aeruginosa. Gene 1994, 148(1):81-86.

43. Hoang TT, Karkhoff-Schweizer RR, Kutchma AJ, Schweizer HP: A broad-host -range Flp-FRT recombination system for site-specific excision of chromosomally-located DNA sequences: application for isolation of unmarked Pseudomonas aeruginosa mutants. Gene 1998, 212(1):77-86.

44. Yeung AT, Bains M, Hancock RE: The sensor kinase CbrA is a global regulator that modulates metabolism, virulence, and antibiotic resistance in Pseudomonas aeruginosa. J Bacteriol 2011, 193(4):918-931.

45. Fey P, Kowal AS, Gaudet P, Pilcher KE, Chisholm RL: Protocols for growth and development of Dictyostelium discoideum. Nat Protoc 2007, 2(6):1307-1316.

46. Amiel E, Acker JL, Collins RM, Berwin B: Uncoupling scavenger receptor Amediated phagocytosis of bacteria from endotoxic shock resistance. Infect Immun 2009, 77(10):4567-4573.

47. Sulston J, Hodgkin J: The Nematode Caenorhabditis elegans. Wood: W. B; 1988.

48. Stiernagle T: Maintenance of C. elegans. In C. elegans. A practical approach. Edited by Hope IA. Oxford, United Kingdom: Oxford University Press; 1999:51-67.

49. Blier AS, Veron W, Bazire A, Gerault E, Taupin L, Vieillard J, Rehel K, Dufour A, Le Derf $F$, Orange $N$, et al: C-type natriuretic peptide modulates quorum sensing molecule and toxin production in Pseudomonas aeruginosa. Microbiology 2011, 157(Pt 7):1929-1944.

50. Wiegand I, Hilpert K, Hancock RE: Agar and broth dilution methods to determine the minimal inhibitory concentration (MIC) of antimicrobial substances. Nat Protoc 2008, 3(2):163-175.

51. Friedman L, Kolter R: Genes involved in matrix formation in Pseudomonas aeruginosa PA14 biofilms. Mol Microbiol 2004, 51(3):675-690.

52. Marr AK, Overhage J, Bains M, Hancock RE: The Lon protease of Pseudomonas aeruginosa is induced by aminoglycosides and is involved in biofilm formation and motility. Microbiology 2007, 153(Pt 2):474-482.

doi:10.1186/1471-2180-13-77

Cite this article as: Neidig et al:: TypA is involved in virulence, antimicrobial resistance and biofilm formation in Pseudomonas aeruginosa. BMC Microbiology 2013 13:77.

\section{Submit your next manuscript to BioMed Central and take full advantage of:}

- Convenient online submission

- Thorough peer review

- No space constraints or color figure charges

- Immediate publication on acceptance

- Inclusion in PubMed, CAS, Scopus and Google Scholar

- Research which is freely available for redistribution 